\title{
LAS COMPETENCIAS DEL PROFESOR EN EL ESPACIO EUROPEO DE EDUCACIÓN SUPERIOR
}

\author{
Professor's Competences on the European Higher Education Space
}

\author{
Emilio García García ${ }^{1}$ \\ Lourdes Martínez Pérez ${ }^{2}$ \\ Elizabeth Carvalho da Veiga ${ }^{3}$
}

\section{Resumen}

En este trabajo analizamos las características de la profesión de profesor, así como algunos obstáculos para su desarrollo profesional. Estudiamos las competencias que se le exigen en las sociedades actuales. Comentamos estudios sobre la formación del profesor en el Espacio Europeo de Educación Superior. Proponemos un modelo reflexivo y crítico (metacognitivo) para el análisis y desarrollo de las competencias del profesorado.

Palabras-clave: Competencias del profesor; Profesión de profesor; Desarrollo profesional; Espacio Europeo de Educación Superior; Proyecto tuning.

\section{Abstract}

This work analyses the professor profession characteristics, like some obstacles to its professional development. The competences required by the modern society, were studied. It discusses the studies about the professor education on the European Higher Education space. A reflexive and critical model (metacognitive) is proposed, for the analysis and development of the professor competences.

Keywords: Competences; Profession; Professional Development; Higher Education; Proyecto tunning.

\footnotetext{
Doutor em psicologia e professor na Universidad Complutense de Madrid. Calle Puerto De Los Leones N.13. Madrid, Espanha. e-mail:garmi@correo.cop.es

Doutora em psicologia e professora na Universidad Complutense de Madrid. Madrid, Espanha. e-mail: lourde@smp.cop.es

Doutora em psicologia e professora da Pontificia Universidade Católica do Paraná. Curitiba, PR. e-mail: bethveiga@hotmail.com
} 


\section{Introducción}

Los profesores pertenecen a una categoría profesional muy heterogénea. Los profesores de educación infantil, primaria, secundaria, universidad, adultos, educación especial, presentan diferencias en formación, reclutamiento, salarios, reconocimiento social, autonomía profesional. Por otra parte, se constatan diferencias en los profesores según países, nivel de desarrollo y culturas. Pero la profesión de profesor también conlleva unas características propias.

La profesión de profesor exige dominar un conjunto de conocimientos y competencias; que ha de aprender en instituciones superiores de formación universitaria; que requiere continua formación y actualización de conocimientos y técnicas; que ha de poner en práctica según principios éticos; para responder a una necesidad personal y social de primer orden, como es la educación.

El profesor ejerce su profesión, normalmente durante muchos años, más de treinta en muchos casos. Durante un periodo tan dilatado, ocurren muchos acontecimientos en su vida y en su entorno. En su ciclo vital, se acumulan experiencias, se amplían conocimientos, se modifican actitudes y valores. En su entorno ocurren cambios científicos, tecnológicos, económicos, sociales y culturales, que alteran radicalmente las demandas al sistema educativo y a su función profesional A la vez, los alumnos presentan características diferenciales y cambiantes en nivel económico y cultural de la familia, capacidades mentales, motivaciones e intereses.

\section{Profesión de profesor y desarrollo profesional}

Desde posturas críticas, sociológicas y pedagógicas, se ha cuestionado las reivindicaciones profesionalizadoras de los profesores, por cuanto conllevan intereses personales y sectoriales elitistas y burocráticos, que serían incompatibles con los ideales democráticos de solidaridad, autonomía y difusión del saber (Burbules y Desmore, 1992). También Apple $(1986,1989)$ denuncia los intereses que operan bajo la reivindicación de una tecnificación de la enseñanza. Pero sería un error identificar profesionalización con tecnificación de los procesos educativos, pues cabe entender la profesión como proceso, como desarrollo de una cultura profesional que avance en niveles de formación, autonomía, responsabilidad y compromiso social en el trabajo (Fernández Pérez, 1988; García García, 1986; 1996; 2006; Ortega y Valera, 1991).

Cabe diferenciar dos tendencias de análisis en la profesionalización del docente: la que argumenta que reforzar la profesión conlleva tecnificación, burocracia, elitismo y control social; y la que, por el contrario, defiende un concepto de profesión históricamente contextualizado, crítico y democrático, y propone avanzar en el desarrollo de una nueva cultura profesional para una educación de calidad. El concepto neoliberal de profesión queda superado por una acepción más social, democrática, histórica y crítica.

Las exigencias de la profesión docente se pueden identificar en cinco ámbitos:

- SABER: conjunto estructurado de conocimientos sobre el mundo natural y sociocultural. Las disciplinas del currículo. Competencias cognitivas;

- HACER: conjunto de procedimientos y estrategias. Competencias procedimentales

- QUERER: motivación, compromiso, esfuerzo. Competencias afectivas;

- CONVIVIR: capacidad para trabajar y relacionarse con compañeros y alumnos. Competencias comunicativas;

- SER: desarrollo del profesor como persona. Responsabilidad ética ante los alumnos, familias y sociedad. Competencias personales (Delors, 1996).

La reivindicación de la profesionalidad por parte de los profesores no puede quedar limitada a aspectos técnicos de la función docente, sino que se ha de plantear un desarrollo profesional más amplio, una nueva cultura profesional, que propicie espacios de reflexión, de crítica sobre las funciones de la educación, modelo de sociedad y proyecto de hombre, procesos de formación, condiciones laborales, variables del proceso enseñanza-aprendizaje, teniendo como objetivo el profesor investigador de su práctica profesional. Algunos autores han diferenciado entre profesionalidad restringida o limitada y profesionalidad desarrollada o ampliada. Se contraponen las características de una y otra en la Tabla 1. (Hoyle, 1974; Stenhousse, 1982; 1987; Giroux, 1987; Kemmis, 1988; Schon, 1992; Imbernon, 1994). 


\section{Tabla 1 - Características de la profesionalidad restringida y desarrollada}

\begin{tabular}{|c|c|}
\hline Profesionalidad restringida & Profesionalidad desarrollada \\
\hline $\begin{array}{l}\text { Destrezas profesionales a partir de la } \\
\text { príctica }\end{array}$ & $\begin{array}{l}\text { Competencias derivadas de una reflexión } \\
\text { entre práctica y teoria }\end{array}$ \\
\hline $\begin{array}{l}\text { Perspectivas limitadas a lo inmediato y } \\
\text { concreto }\end{array}$ & $\begin{array}{l}\text { Perspectivas que comprenden el contexto } \\
\text { social de la educación }\end{array}$ \\
\hline $\begin{array}{l}\text { Sucesos y experiencias del aula, } \\
\text { considerados aisladamente }\end{array}$ & $\begin{array}{l}\text { Valoración de las experiencias del aula, } \\
\text { desde objetivos y metas más amplios }\end{array}$ \\
\hline Metodologia introspectiva e intuitiva & $\begin{array}{l}\text { Metodología basada en la reflexión crítica } \\
\text { a partir de la práctica, y en diálogo con } \\
\text { compañeros }\end{array}$ \\
\hline $\begin{array}{l}\text { Valoración de la autonomia profesional } \\
\text { Limitada participación en actividades } \\
\text { profesionales }\end{array}$ & $\begin{array}{l}\text { Valoración de la colaboración profesional } \\
\text { Alta participación en actividades } \\
\text { profesionales }\end{array}$ \\
\hline Poca lectura de literatura profesional & Lectura regular de literatura profesional \\
\hline $\begin{array}{l}\text { Participación en formación de cursos } \\
\text { prácticos }\end{array}$ & $\begin{array}{l}\text { Participación en tareas de formación de } \\
\text { naturaleza más teórica }\end{array}$ \\
\hline $\begin{array}{l}\text { Modelo técnico. Racionalidad técnica. } \\
\text { Modelo Proceso-Producto }\end{array}$ & $\begin{array}{l}\text { Modelo reflexivo - creativo - crítico. El } \\
\text { profesor como investigador en el aula }\end{array}$ \\
\hline
\end{tabular}

Diversos informes de la OCDE han señalado, como características del buen profesor, las siguientes:

- compromiso con el trabajo. Es condición determinante para la buena valoración por los alumnos y el éxito en la tarea;

- afectividad con los alumnos. Un comportamiento afectivo hacia los alumnos, con empatía, optimismo, estima y apoyo, son cualidades valoradas muy positivamente;

- buen conocimiento de la materia que enseña y empleo de técnicas didácticas adecuadas. Saber lo que se enseña y enseñarlo bien. Combinar metodologías variadas, partiendo de las características diferenciales de los alumnos y del grupo;
- Trabajo colaborativo en grupo de profesores. El trabajo en grupo es altamente valorado, aunque no tan practicado. Analizar las experiencias y dificultades en sesiones formales, pero también en conversaciones de pasillo, es requisito para mejorar las competencias del profesor;

- Pensamiento reflexivo y crítico. Reflexionar en la práctica y sobre su práctica, a efectos de poner en juego nuevas hipótesis y desarrollar teorías y adoptar nuevas estrategias didácticas;

- Motivación por la calidad. Los buenos profesores se comprometen con la innovación y la calidad en los centros, procurando proyectos compartidos, liderazgos eficaces, apertura al contexto comunitario (Hargreaves, 1992; 1996; 
Marchesi y Martin, 2000). En el desarrollo profesional del profesor distinguimos los siguientes componentes:

A) Metas. El desarrollo profesional está en estrecha dependencia de los valores, los objetivos, las expectativas que un profesor mantiene en el ejercicio de su profesión. Tales metas admiten modulaciones bien distintas: desde entender la educación como transmisión de conocimientos de las diversas disciplinas del curriculum, hasta propiciar la socialización de los alumnos y el comportamiento solidario; desde la preocupación por el desarrollo personal y la formación integral, hasta la igualdad de oportunidades de los alumnos y el tratamiento equitativo; desde la implicación emocional fuerte en su profesión, con los costes que ello implica, hasta considerarla como un medio de vida. En alguna medida todos los profesores comparten estas metas, pero el mayor o menor peso que les conceden unas u otras llevarán a comportamientos y valoraciones bien diferenciados.

B) Exigencias conflictivas de la profesión. La profesión docente está profundamente afectada por los cambios científicos, tecnológicos, económicos, sociales, políticos y culturales habidos en la sociedad. Las críticas a la educación y a la función del profesor son una expresión de la puesta en cuestión de la sociedad y cultura contemporánea. A la escuela se le exige hoy demandas encontradas y crecientes por parte de los alumnos, las familias, las fuerzas sociales, la administración. El profesor se encuentra en el centro del conflicto ante exigencias cada vez mayores y conflictivas, hasta el punto de vivir situación de alto riesgo con consecuencias para su salud. Ha de ser transmisor y a la vez crítico de la cultura ante las nuevas generaciones; ha de enseñar a pensar, a decidir, a disfrutar del ocio, a cuidar de la salud, a respetar el medio natural y el patrimonio sociocultural, a ser solidario con los demás. Se le pide integrar en la sociedad al alumno como miembro comprometido y responsable, pero se le presenta una sociedad en continua crisis económico-social, con paro, competitividad, injusticia y marginación. Se le pide una relación personal con el alumno, un trabajo en equipo, una permanente actualización en su quehacer. Se pretende un profesor agente compensador de las desigualdades sociales y de las deficiencias personales, al integrar a alumnos con necesidades educativas especiales en una escuela inclusiva, para todos.
Son numerosos los estudios que constatan la insatisfacción personal, el estrés y las patologías vinculadas a la profesión docente. Los sindicatos y asociaciones de profesores constatan los riesgos de la profesión para la salud y reclaman programas de intervención. Son muchos los profesores quemados y con patologías más graves. Entre otras causas se señalan especialmente (García García, 1988; 1996):

- el cuestionamiento continuo de la profesión y de las reformas educativas;

- la pérdida de estatus, prestigio y consideración social;

- las exigencias crecientes y contradictorias, junto con la falta de apoyos e información;

- salarios bajos, condiciones laborales inadecuadas, escasa promoción;

- vulnerabilidad y soledad ante alumnos, padres, compañeros e inspección;

- problemas de conducta y disciplina en las aulas;

- presión asfixiantes de programaciones, evaluaciones, reuniones múltiples.

A los profesores se les responsabiliza del éxito - fracaso en el aprendizaje de los alumnos, pero no se les proporcionan los apoyos necesarios, como formación, equipamientos, materiales, incentivos. Se ha ampliado la escolaridad obligatoria de la población, lo que conlleva más heterogeneidad del alumnado y porcentaje alto de desmotivados. A la diversidad de capacidades de los alumnos que requieren atenciones educativas específicas, se añaden la diversidad de etnias, culturas, lenguas que traen consigo la inmigración. El nivel económico de los alumnos es una variable importantísima, pero que está fuera del control del profesor. Asimismo el profesor ha de competir con otros agentes de socialización, con extraordinario poder y direcciones encontradas, como son los medios de masas, los iguales, Internet.

El profesor tradicionalmente ha sido depositario y transmisor de conocimientos con reconocimiento y autoridad de ascendencia, pero hoy se le exige un nuevo rol de negociación, diálogo, consenso, desde una cultura de participación. Se demanda una enseñanza comprensiva para todos desde el valor de una escuela inclusiva y de calidad, lo que puede resultar problemático. La participación en los proyectos de centro, y a la vez la participación 
en las actividades de la comunidad, requieren dedicar en el profesorado recursos extra.

C) Formación inicial y permanente.

Los profundos cambios en el entorno económico, social y cultural, así como en las características del alumnado, exigen un replanteamiento continuo de la función docente. El profesor demanda una formación inicial que le proporcione las competencias precisas para dar respuesta a los nuevos desafíos, pero más aún necesita una formación continua, ya que sólo desde ella podrá hacer frente con razonable éxito a las demandas conflictivas, y lograr niveles adecuados de desarrollo y satisfacción profesional, superando actitudes negativas ante los cambios continuos en el mundo de la educación.

Los programas de formación han de considerar las diversas funciones que al profesor se le exige. El profesor ha de diseñar el proceso de enseñanza - aprendizaje en el aula, como procurar la adquisición de conocimientos y destrezas en un clima de colaboración, orientar a los alumnos, participar en el funcionamiento del centro, etc. Las competencias del profesor requieren un marco de capacitación idóneo que estimule la reflexión sobre la práctica (Giroux, 1987; Apple, 1986; Schon, 1992; Gimeno, 1986). Ello conlleva principios reguladores como: partir de los conocimientos, necesidades y preocupaciones que los profesores tienen; dialogar y discutir ideas y alternativas en grupo, en un ciclo de acción - reflexión - innovación; valerse de diferentes metodologías, como expertos en solución de problemas, lectura de literatura científica, participación en la dinámica del centro y de la comunidad.

D) Condiciones laborales e incentivos. Los incentivos que los profesores reciben, y las condiciones laborales en las que realizan su trabajo, son determinantes de su satisfacción personal. Si el profesor dispone de condiciones adecuadas, normalmente se sentirá más satisfecho, valorado e implicado en su trabajo. Las condiciones tienen que ver con el número de alumnos por clase, los materiales curriculares y recursos informáticos, espacios adecuados para trabajo individual y grupal, tiempos disponibles para ello. Por otra parte, para tener un clima positivo de trabajo con los compañeros de claustro, resulta crítico el rol de la dirección y el apoyo a las iniciativas innovadoras del profesorado y a la cohesión grupal.

Son también incentivos muy importantes para el trabajo del profesorado y su desarrollo profesional: una carrera docente que resulte atractiva; un salario digno y una posibilidad de promoción. Además de la tradicional remuneración por antigüedad, hay también otros incentivos que pueden jugar un importante papel, como tutoría de nuevos profesores y supervisión de prácticas; responsabilidad en la gestión del centro; participación en programas de innovación e investigaciones.

Deben quedar claros los requisitos y reconocimientos asociados al incremento salarial. Para la valoración del trabajo de los profesores son cuestiones clave: quien valora y a quien, qué valorar y para qué. La participación de un equipo de evaluadores, con la implicación de compañeros del centro, resulta a priori el más idóneo, pero es un procedimiento costoso y con serios inconvenientes. Evaluar sistemáticamente a todo el profesorado es complicado, y sobre todo requiere tener claro una cuestión previa: si la evaluación se considera como ayuda y recurso al profesor para su mejora, o si lleva implicadas mejoras salariales. Una evaluación que no lleve pareja un incentivo salarial no tiene la necesaria aceptación. Por ello, una estrategia puede ser limitar la evaluación cuando se opta voluntariamente a un incentivo profesional determinado.

En cuanto a qué evaluar, hay cierto consenso en que al menos deben considerarse cuatro aspectos de la práctica: la competencia en el proceso de enseñanza-aprendizaje en el aula; la función de tutoría y orientación individual a los alumnos; la participación en el funcionamiento del centro, y la implicación en las actividades de la comunidad y el contexto sociocultural. Pero las técnicas para recoger información y valorarla no resultan fáciles, especialmente de la vida interna del aula, dada la poca disponibilidad que los profesores damos a la observación de "nuestra" clase por colegas.

E) Cultura profesional. La cultura de los profesores y los centros, así como la calidad profesional, son temas de investigación de gran peso en la actualidad. Hargreaves (1992; 1996) ha diferenciado en la cultura dos componentes: el contenido y la forma. Por contenido entiende los conocimientos, sentimientos y comportamientos que en alguna medida comparten los profesores, los valores y creencias, las expectativas y metas, las actitudes y motivaciones. La forma hace referencia a los sistemas de organización e integración que se establecen en los centros, y que son responsables, en gran medida, del clima laboral y satisfacción personal y profesional. Diferencia cinco formas de cultura: 
a) Individualismo. La cultura individualista está profundamente arraigada en el profesorado. El profesor se percibe como responsable único de la enseñanza, quizá no tanto del aprendizaje, de "su" materia, a "su" clase. Es muy celoso de su autonomía como docente, y paralelamente reacio a observaciones, evaluaciones y controles. Tiene el riesgo del aislamiento y empobrecimiento de las relaciones con colegas, pero también una autoestima y valoración de su competencia como profesor.

b) Fragmentación. Se caracteriza porque los profesores, en pequeños grupos, se enfrentan entre ellos, imposibilitando la cooperación en un trabajo colectivo. Ello viene fomentado por la estructura y organización de los centros en departamentos y áreas separadas, así como el supuesto prestigio y valoración de las asignaturas.

c) Agrupamiento obligado. Caracterizada por una autoridad educativa que impone proyectos comunes, y hasta planes de mejora, pero que no goza de la aceptación del profesorado, que los ve como imposición, obstaculizando la implicación personal.

d) Colaboración. Se comparten básicamente las metas y valores educativos, respetando las individualidades y discrepancias. La colaboración va más allá de la acción docente en la vida del centro, y abarca las relaciones personales, ocio compartido. En una cultura de colaboración, además de la función clave de la dirección, está presente una alta motivación e implicación en la enseñanza. Para enfrentarse a los nuevos retos en las escuelas, se requiere superar la separación existente entre departamentos, hacia modelos más flexibles, que permitan ajustes a los cambios continuos que se dan en la educación. Además de las clásicas estructuras verticales y jerárquicas, se necesitan redes de comunicación más flexibles y horizontales. Una cultura de colaboración favorece el bienestar en el trabajo y es condición necesaria para una educación de calidad y una escuela inclusiva. La forma como los alumnos pueden aprender las competencias de solidaridad, respeto, comportamiento cívico, requiere ese mismio clima propicio.

\section{Enseñanza-aprendizaje de competencias}

En los procesos de reforma de la educación, es muy frecuente concentrar interés y recursos en reformar los planes de estudio, entendiendo por tal las asignaturas a impartir en el currículo, los programas y la selección de los contenidos, dada la acumulación progresiva de los conocimientos. Pero en los procesos de enseñanzaaprendizaje, además de los contenidos, no es menos importante la forma de enseñarlos y aprenderlos, los procedimientos y la metodología. Estas formas de aprender son estrechamente dependientes de las formas de evaluar. Este saber cómo o conocimiento procedimental es tan necesario cómo el saber qué o conocimiento declarativo, especialmente en la vida profesional y personal.

En la sociedad del conocimiento se plantean nuevas exigencias. Son necesarias, pero no suficientes, las enseñanzas y aprendizajes tradicionales: presentar al alumno los contenidos de las asignaturas, el conjunto de los conocimientos más relevantes de forma clara y sistemática, y con recursos que resulten interesantes y faciliten el aprendizaje, como transparencias, video, ordenador, internet, etc. También son necesarios sistemas de evaluación del aprendizaje del alumno y también de la actuación del profesor, así como de los procesos y resultados alcanzados por la institución en sus diversos niveles y dimensiones.

Por otra parte, no parece que los exámenes tradicionales, parciales y finales, sean el único y menos el mejor procedimiento para garantizar y acreditar los aprendizajes del alumno, dejando así tranquilo al profesor, la institución, los padres, la sociedad. Reconocer o recordar por escrito algunas informaciones que ha transmitido el profesor en las clases y recogido el alumno en sus apuntes pueden dar cuenta de un tipo de memoria del alumno, pero no parece suficiente garantía de que haya comprendido, asimilado, integrado en su mente los conocimiento, de modo que pueda recuperarlos y utilizarlos cuando sea preciso para resolver los problemas más allá del ámbito académico. En la vida diaria, en los contextos profesionales, no parece que sea prioritario responder al tipo de preguntas que se formulan en los exámenes. Muy al contrario se requiere ir más allá de la información dada, seguir aprendiendo continuamente, identificar lo que es preciso aprender, dónde y cómo encontrar los recursos para el aprendizaje, saber identificar los problemas que merece la pena resolver, aprender a trabajar con otros en equipos eficaces, aprender a convivir, etc.

Los profesores dedicamos gran parte de nuestro tiempo y energía a ampliar nuestros conocimientos de la asignatura para estar al día, a preparar las clases. Cada curso procuramos mejorar 
los materiales, esquemas, gráficos, problemas, incluso organizamos seminarios, grupos de discusión, sesiones interactivas, estudios de casos, etc. Procuramos suscitar y mantener la atención e interés de nuestros alumnos en la clase, lamentamos que tras tanta inversión en tiempo y recursos a la tarea docente los resultados sean tan limitados. Seguimos instalados en los supuestos tradicionales de que basta con enseñar bien los contenidos para que el alumno los aprenda. Pero el alumno aprende cuando la información le interesa, es significativa cognitiva y emocionalmente, conecta con preocupaciones personales o profesionales, con las demandas de la realidad fuera del aula, y se compromete con su propio proceso de formación. Las competencias para un aprendizaje autónomo y continuo a lo largo de la vida es un tipo de aprendizaje procedimental que exige práctica y no sólo clases magistrales de conocimiento declarativo.

Un objetivo prioritario de la educación debe ser capacitar a sus alumnos para el mayor desarrollo personal y ello requiere conocimientos, sentimientos y comportamientos responsables y solidarios. Los conocimientos y procedimientos, las memorias declarativas y procedimentales que un profesor tiene sobre su especialidad no se transfiere a la mente de sus alumnos con solo explicarlas en clase, aunque sea de la forma más atractiva y estimulante imaginable. El estudiante aprenderá si asimila los conocimientos, los hace suyos, los integra, los experimenta relevantes en su vida personal y profesional, los utiliza para identificar y resolver problemas. Como exigencias para este tipo de aprendizaje señalamos (García García, 2004):

Autoconocimiento. Conocimiento de uno mismo de sus potencialidades y sus déficits, reflexión sobre sus puntos fuertes y sus debilidades. Conocerse para elaborar su proyecto profesional y personal.

Automotivación. Compromiso e implicación en el proyecto personal de formación. Las fuentes de la motivación pueden ser internas como la propia satisfacción con lo que se hace, el deseo de saber y hacer las cosas bien, la superación personal; o bien externas, como reconocimiento social, calificaciones, recompensas monetarias etc. Lo mas adecuado es combinar en proporción razonable las dos fuentes de motivación.

Autoestima. Autoconfianza y autoeficacia, es decir, considerarse capaz de alcanzar las metas que se propone. Creencia en las propias competencias para afrontar las exigencias de las situación y resolver los problemas.
Autocontrol. Controlar las respuestas impulsivas, disponer de un tiempo para evaluar la situación, las demandas que se plantean, las alternativas posibles, los recursos que se exigen, los pros y contras de las acciones a tomar.

Autonomía. Responsabilidad y solidaridad. Libertad de pensamiento, sentimiento, imaginación para dar sentido a su vida, y alcanzar las cotas más altas de bienestar y felicidad, y a la vez compromiso con el desarrollo humano global.

Atribución causal equilibrada. La tendencia sesgada a considerarse responsable de los éxitos o de los fracasos es tan inapropiada como el responsabilizar siempre a los demás. Así mismo, en ocasiones puede ser la suerte, la dificultad de la tarea, el esfuerzo invertido, etc, las causas del éxito o fracaso.

Convivencia. Aprender a convivir en los diferentes y simultáneos espacios en los que transcurre nuestra vida: escolar, familiar, laboral, sociocultural es muy importante y urgente. Aprender a trabajar con otros compartiendo y potenciando nuestras competencias y superando limitaciones. La educación ha de mostrar la complejidad y diversidad de los seres humanos, a la vez que sus semejanzas y mutuas interdependencias en un mundo globalizado.

Constancia. Perseverancia y esfuerzo. Resistir las frustraciones que conllevan los fracasos y perseverar en la tarea de modo razonable para alcanzar resultados, mantener la concentración y tensión en la meta siendo capaz de aplazar gratificaciones y recompensas. Completar las tareas exige superar las dificultades personales y los obstáculos exteriores.

Creatividad. Descubrir, buscar, formular y solucionar problemas valiosos. No se trata solo de saber resolver problemas, sino de discernir qué problemas merece la pena abordar.

Iniciativa. Motivación de logro y no tanto miedo al fracaso. El temor a fracasar puede bloquear empresas y proyectos. Cometer errores, equivocarse, no es fracasar. Aprender de los errores es ser inteligente. En las tareas y objetivos de dificultad moderada es donde la persona motivada por el éxito más se implica.

Pensamiento analítico, creativo y práctico. Hay momentos y contextos donde es mejor ser analítico, otras creativo o más práctico. Es importante discernir cuándo aplicar esas habilidades mentales según las características del medio.

Pensamiento reflexivo. La mente humana tiene capacidades metacognitivas, de reflexión, de volver 
sobre sí misma y tomarse como objeto de conocimiento y mejora. Conocer sobre los procesos cognitivos, motivacionales, emocionales, sobre las propias capacidades y limitaciones, sobre los comportamientos y resultados, es la conquista más adaptativa de la mente humana.

Aprender sobre el conocer, querer, sentir, actuar no es cuestión solo teórica, sino de gran relevancia práctica, especialmente en aquellas profesiones, como la de profesor, cuyo quehacer exige continua relación con otras personas, con otras mentes, pensamientos, sentimientos y comportamientos. Para mejorar la práctica docente hacia una enseñanza y aprendizaje de más calidad es preciso que los profesores prestemos especial atención a nuestra mente, nuestros pensamientos, sentimientos y comportamientos, a la vez que nos interesamos en la de nuestros alumnos. En la interacción educativa aprenden y enseñan profesores y alumnos. Unos y otros desarrollan competencias de carácter cognitivo, comunicativo, afectivo, personal y social. El desafío es interno, personal de cada uno de nosotros: la necesidad de educarnos para educar, de cambiar nuestra mentalidad, nuestra forma de pensar, sentir y comportarnos.

\section{Competencias del estudiante universitario}

El "Proyecto Tuning" (2003) supone un avance muy importante en el estudio de las diversas y complejas competencias que hoy se demandan a la educación en los distintos niveles, particularmente en el nivel universitario. El ECTS es la unidad de medida que regirá el sistema académico. A diferencia de los actuales créditos, que se limitan a computar solamente las horas lectivas, el ECTS reglamenta la cantidad de trabajo que tiene que llevar a cabo el estudiante para superar los estudios. Establece las horas de enseñanza prácticas y teóricas, pero también las horas de estudios, la elaboración de trabajos, de consultas bibliográficas, de tutorías, etc. Cada curso los estudiantes tendrán que realizar sesenta créditos europeos. Un crédito establece entre veinticinco y treinta horas.

Cien instituciones universitarias, representativas de la Unión Europea, han participado en la primera fase del proyecto (20002002), que ha sido coordinado por las Universidad de Deusto (España) y Groningen (Holanda). El
Proyecto Tuning se apoya en anteriores experiencias de cooperación, realizadas en el marco de los proyectos de redes temáticas de Sócrates-Erasmus, y de los proyectos piloto ECTS. Se escogió el término Tuning, afinar en términos musicales, con la intención de transmitir la idea no de unificar y obligar a homogeneizar, sino de sintonizar y converger. El Proyecto Tuning persigue ante todo proteger la diversidad de la educación europea, desde el máximo respeto a la autonomía de las instituciones. El Proyecto se propone determinar puntos de referencia para las competencias genéricas que los estudiantes han de adquirir por el hecho de ser universitarios, y las específicas de cada disciplina o campo temático. Las competencias describen los resultados del aprendizaje, esto es, lo que un estudiante sabe y puede demostrar una vez completado su proceso de aprendizaje. Los académicos, los estudiantes y los empleadores han sido consultados sobre las competencias que se desea adquieran los titulados.

El Proyecto Tuning se ha elaborado con una metodología basado en cuatro ejes: 1. Competencias genéricas; 2. Competencias específicas; 3. Papel del sistema ECTS en el currículo; 4. La función del aprendizaje, la docencia, la evaluación y el rendimiento en relación con el aseguramiento y evaluación de la calidad. Durante la primera fase del Tuning se ha puesto especial énfasis en los tres primeros ejes, y el cuarto se ha trabajado en la segunda parte del Proyecto, 20032004. El objetivo más general del Proyecto Tuning es servir de plataforma para el intercambio de experiencias y conocimientos entre países e instituciones de educación superior, con el fin de facilitar una mayor transparencia de las estructuras educativas, impulsando la innovación y la calidad.

El Informe Tuning (2003) diferencia tres grandes ámbitos de competencias o destrezas: a) conocer o comprender: el conocimiento teórico de un campo académico, la capacidad de conocer y comprender; b) saber cómo actuar: la aplicación practica y operativa del conocimiento a ciertas situaciones; c) saber cómo ser: los valores como parte integrante de la forma de percibir a los otros y vivir en un contexto social. Las competencias representan una combinación de atributos (con respecto al conocimiento y sus aplicaciones, aptitudes, destrezas y responsabilidades) que describen el nivel o grado de suficiencia con que una persona es capaz de desempeñarlos. 
El concepto de competencia hace referencia a las capacidades, aptitudes, destrezas, recursos que permiten a una persona realizar una acción o un producto. Poseer una competencia significa que una persona, al manifestar una cierta capacidad o destreza al desempeñar una tarea, puede demostrar que la realiza de manera tal que permita evaluar el grado de realización de la misma. Las competencias se sitúan en un continuo y cada persona puede dominar unas u otras (Arguelles, 1997; Levy-Leboyer, 2000).

El Proyecto Tuning analiza dos conjuntos de competencias. Las genéricas y las específicas. Las competencias específicas se relacionan con cada área temática y resultan claves para cada titulación universitaria, ya que están específicamente relacionadas con el conocimiento concreto de un área temática. Son pues las competencias relacionadas con las disciplinas académicas y son las que confieren identidad y consistencia a cualquier programa. Las competencias genéricas son las que se consideran que cualquier estudiante universitario debe adquirir por el hecho de pasar una universidad. Son aquellas que cualquier titulación debe proporcionar, tales como capacidad de aprender, de analizas y síntesis, etc

El Proyecto Tuning limita el estudio a 30 competencias genéricas clasificadas en tres grupos:

a) competencias instrumentales, que incluyen destrezas cognitivas, como la capacidad de comprender y manipular ideas y pensamientos; capacidades metodológicas como tomar decisiones y resolver problemas: capacidades tecnológicas como la capacidad de manejar ordenadores; y capacidad lingüística, como comunicación oral y escrita o conocimiento de una lengua.

b) competencias interpersonales: capacidades individuales relativas a la capacidad de expresar los propios sentimientos, habilidades críticas y de autocrítica. Destrezas sociales relacionadas con las relaciones interpersonales, la capacidad de trabajar en equipo, el compromiso social y ético. Estas competencias facilitan la interacción y cooperación social.

c) competencias sistémicas: son las destrezas y habilidades que conciernen a los sistemas como totalidad. Suponen una combinación de la comprensión, la sensibilidad y el conocimiento que permiten al individuo ver como las partes de un todo se relacionan y agrupan. Estas capacidades incluyen la habilidad de planificar cambios para mejorar e innovar los sistemas. Las competencias sistémicas requieren como base la adquisición previa de competencias instrumentales e interpersonales.

Como conclusiones del primer informe del Proyecto Tuning, sobre la reflexión y el debate de las competencias, destacamos:

- El desarrollo de las competencias sintoniza con el principio regulador de poner el énfasis, no tanto en la enseñanza del profesor, cuanto en el aprendizaje del alumno. Es el estudiante quien tiene que aprender, y el profesor ha de facilitar, orientar y promover tales aprendizajes. Los aprendizajes no quedan limitados al contenido del currículum, sino que incorporan las destrezas, las competencias, las motivaciones y las aptitudes;

- La identificación y el desarrollo de las competencias va estrechamente ligado a los perfiles académicos y profesionales. Los títulos de grado han de posibilitar salidas profesionales y han de estar caracterizados por un conjunto de competencias para ello. La definición de las competencias requeridas facilita los procesos de evaluación y calidad, al identificar objetivos, recursos, procesos y resultados;

- La reflexión y el análisis crítico de las competencias específicas de cada área temática permitirá desarrollar titulaciones comparables y comprensibles en el Espacio Europeo de Educación Superior (EEES) y facilitará la movilidad de estudiantes, profesores y graduados;

- Las competencias no deben quedar acotadas a las demandas económicas y sociales del momento, sino que se ha de promover la reflexión crítica sobre lo dado, y las alternativas de mejora. La universidad tiene como dimensión esencial la crítica de la cultura y la propuesta razonada y razonable de proyectos alternativos. Los estudiantes exigen cualificaciones idóneas para el mercado laboral, pero la universidad no puede quedar reducida a satisfacer las demandas del mercado y los intereses de grupos sociales y profesionales; 
- El Proyecto Tuning ha consultado a graduados, empleadores y académicos, respecto a las competencias genéricas requeridas en la sociedad del conocimiento, y es de destacar los acuerdos y también las discrepancias en las ponderaciones de unos y otros, como se puede visualizar en la Tabla 2.

Hay que notar que en líneas generales se valora como competencias más importantes: la capacidad de análisis y síntesis; la capacidad de aprender, la capacidad de resolver problemas; la capacidad para aplicar conocimientos en la práctica; la capacidad para adaptarse a nuevas situaciones; la preocupación por la calidad; las habilidades de gestión de la información; la capacidad para trabajar de forma autónoma; y el trabajo en equipo.

En el otro extremo de competencias menos valoradas están: conocimientos de culturas y costumbres de otros países; apreciación de la diversidad y multiculturalidad; habilidad para trabajar en un contexto internacional; liderazgo; habilidades de investigación; habilidades para diseño y gestión de proyectos; y conocimiento de una segunda lengua.

La discrepancia en las apreciaciones entre los tres grupos (académicos, graduados y empleadores) especialmente está en: la competencia de conocimientos generales básicos, que para graduados y empleadores está entre los niveles 20 y 21 , mientras que para los académicos parece en primer lugar. Las competencias en la computación es considerado más importante por los graduados, el puesto 7 de 30 , menos por los empleadores y profesores, 17 y 16. Las destrezas interpersonales, en general tienden a ser clasificadas más bajas por los académicos que por los graduados y empleadores. La mayoría de las competencias que aparecen en la posición más alta de la escala son las instrumentales y las sistémicas.

\section{Tabla 2 - Competencias Genéricas del estudiante universitario. Valoración por titulados, empleadores y profesores universitarios}

\begin{tabular}{|c|c|c|c|c|}
\hline & \multirow[b]{2}{*}{$\begin{array}{l}\text { COMPETENCIAS GENÉRICAS } \\
\text { del estudiante universitario }\end{array}$} & \multicolumn{3}{|c|}{ Orden de importancia } \\
\hline & & $\begin{array}{l}\text { Gradua- } \\
\text { dos }\end{array}$ & $\begin{array}{l}\text { Emplea- } \\
\text { dores }\end{array}$ & $\begin{array}{l}\text { Profe- } \\
\text { sores }\end{array}$ \\
\hline \multirow{10}{*}{ 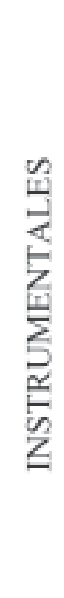 } & Capacidad de análisis y sintesis & $1 / 30$ & $3 / 30$ & $2 / 17$ \\
\hline & Planificación y gestión del tiempo & $10 / 30$ & $13 / 30$ & - \\
\hline & Conocimientos generales sobre el área de estudio & $20 / 30$ & 21730 & $1 / 17$ \\
\hline & Conocimientos básicos de la profesión & $19 / 30$ & $23 / 30$ & $8 / 17$ \\
\hline & Comunicación oral y escrita en la propia lengua & $12 / 30$ & $11 / 30$ & 9717 \\
\hline & Conocimiento de una segunda lengua & $24 / 30$ & $26 / 30$ & \\
\hline & Habilidades básicas de manejo de ordenador & $7 / 30$ & $17 / 30$ & $16 / 17$ \\
\hline & Habilidades de gestión de la información & $5 / 30$ & $8 / 30$ & - \\
\hline & Resolución de problemas & $2 / 30$ & $4 / 30$ & $\ldots$ \\
\hline & Toma de decisiones & $15 / 30$ & $15 / 30$ & $12 / 17$ \\
\hline
\end{tabular}




\begin{tabular}{|c|c|c|c|c|}
\hline \multirow{8}{*}{ 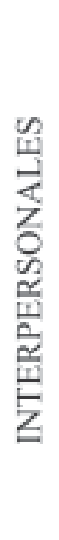 } & Capacidad crítica y autocrítica & $17 / 30$ & $16 / 30$ & $6 / 17$ \\
\hline & Trabajo en equipo & $14 / 30$ & $6 / 30$ & - \\
\hline & Habilidades interpersonales & $9 / 30$ & $9 / 30$ & $14 / 17$ \\
\hline & Capacidad de trabajar en equipo interdisciplinario & $21 / 30$ & $18 / 30$ & $10 / 17$ \\
\hline & $\begin{array}{l}\text { Capacidad para comunicarse con personas no } \\
\text { expertas }\end{array}$ & $18 / 30$ & $20 / 30$ & -. \\
\hline & Apreciación de diversidad y multiculturalidad & $29 / 30$ & $28 / 30$ & $17 / 17$ \\
\hline & Habilidad de trabajar en un contexto internacional & $26 / 30$ & $27 / 30$ & -- \\
\hline & Compromiso ético & $28 / 30$ & $22 / 30$ & $13 / 17$ \\
\hline \multirow{12}{*}{ 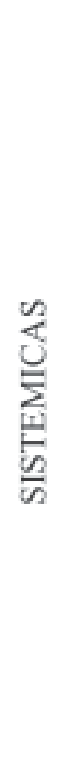 } & Capacidad de aplicar conocimientos en la práctica & $6 / 30$ & $2 / 30$ & $5 / 17$ \\
\hline & Habilidades de investigación & $25 / 30$ & $29 / 30$ & $11 / 17$ \\
\hline & Capacidad de aprender & $3 / 30$ & $1 / 30$ & $3 / 17$ \\
\hline & Capacidad para adaptarse a nuevas situaciones & $8 / 30$ & $7 / 30$ & $7 / 17$ \\
\hline & Capacidad para generar nuevas ideas. Creatividad & $16 / 30$ & $10 / 30$ & $4 / 17$ \\
\hline & Liderazgo & $27 / 30$ & $25 / 30$ & - \\
\hline & $\begin{array}{l}\text { Conocimiento de culturas y costumbres de otros } \\
\text { paises }\end{array}$ & $30 / 30$ & $30 / 30$ & $\ldots$ \\
\hline & Habilidad para trabajar de forma autónoma & $4 / 30$ & $12 / 30$ & -- \\
\hline & Diseño y gestión de proyectos & $23 / 30$ & $24 / 30$ & - \\
\hline & Iniciativa y espiritu emprendedor & $22 / 30$ & $19 / 30$ & $\ldots$ \\
\hline & Preocupación por la calidad & $11 / 30$ & $5 / 30$ & - \\
\hline & Motivación de logro & $13 / 30$ & $14 / 30$ & $\ldots$ \\
\hline
\end{tabular}

\section{Competencias genéricas y especificas del profesor}

En el Libro Blanco Titulo de Grado en Magisterio (ANECA, 2005) se valora la importancia de determinadas competencias, en relación con los perfiles profesionales de las dos titulaciones de magisterio que propone: Educación Infantil y Educación Primaria. En el Titulo de Educación Primaria se proponen cuatro perfiles: Educación física, Lengua extranjera, Educación musical, Educación especial.

$\mathrm{EI}=$ Educación Infantil EP-EF= Educación Primaria, Perfil de Educación Física
EP-LE $=$ Educación Primaria, Perfil de Lengua Extranjera

EP-EM= Educación Primaria, Perfil de Educación Musical

EP-NEE $=$ Educación Primaria, Perfil de Necesidades Educativas Especificas.

Se recogen en la Tabla 3, los valores medios de las competencias transversales dados por 180 académicos de 18 universidades españolas, a quienes se les pidió que valoraran el nivel de competencia profesional con puntuación de 1 (ningún nivel en esa competencia) a 4 (alto nivel en esa competencia). 


\section{Tabla 3 - Competencias genéricas}

\begin{tabular}{|c|c|c|c|c|c|}
\hline $\begin{array}{l}\text { COMPETENCIAS TRANSVERSALES } \\
\text { O GENERICAS }\end{array}$ & $\begin{array}{l}\text { EP- } \\
\text { LE }\end{array}$ & $\begin{array}{l}\text { EP- } \\
\text { EF }\end{array}$ & $\begin{array}{l}\text { EP- } \\
\text { EM }\end{array}$ & $\begin{array}{l}\text { EP- } \\
\text { NEE }\end{array}$ & EI \\
\hline \multicolumn{6}{|l|}{ INSTRUMENTALES } \\
\hline Capacidad de análisis y sintesis & 2,94 & 2,98 & 3,21 & 3,13 & 3,25 \\
\hline Capacidad de organización y planificación & 3,00 & 3,31 & 3,31 & 3,42 & 3,27 \\
\hline $\begin{array}{l}\text { Comunicación oral y escrita en la lengua } \\
\text { materna }\end{array}$ & 3,57 & 3,32 & 3,28 & 3,50 & 3,65 \\
\hline Conocimiento de una lengua extranjera & 3,90 & 2,25 & 2,23 & 2,13 & 2,47 \\
\hline Conocimientos de informática & 2,36 & 2,26 & 2,58 & 2,59 & 2,23 \\
\hline Capacidad de gestión de la información & 2,36 & 2,46 & 2,57 & 2,43 & 2,38 \\
\hline Resolución de problemas & 2,78 & 3,05 & 2,95 & 3,25 & 3,01 \\
\hline Toma de decisiones & 2,75 & 3,12 & 2,97 & 3,04 & 3,01 \\
\hline \multicolumn{6}{|l|}{ PERSONALES } \\
\hline Trabajo en equipo & 2,96 & 3,23 & 3,11 & 3,25 & 3,20 \\
\hline Trabajo en equipo interdisciplinar & 2,85 & 2,89 & 2,87 & 3,07 & 3,04 \\
\hline Trabajo en un contexto internacional & 2,78 & 2,16 & 2,07 & 1,94 & 1,96 \\
\hline Habilidades en las relaciones interpersonales & 3,15 & 3,19 & 3,18 & 3,19 & 3,24 \\
\hline $\begin{array}{l}\text { Reconocimiento a diversidad y multi- } \\
\text { culturalidad }\end{array}$ & 3,18 & 3,09 & 3,11 & 3,38 & 3,25 \\
\hline Razonamiento crítico & 2,97 & 2,82 & 2,92 & 2,75 & 2,92 \\
\hline Compromiso ético & 3,04 & 3,07 & 3,13 & 3,25 & 3,06 \\
\hline \multicolumn{6}{|l|}{ SISTEMICAS } \\
\hline Aprendizaje autónomo & 3,17 & 2,93 & 3,18 & 3,23 & 3,05 \\
\hline Adaptación a nuevas situaciones & 3,01 & 3,07 & 2,94 & 3,40 & 3,31 \\
\hline Creatividad & 2,88 & 3,01 & 3,30 & 3,20 & 3,40 \\
\hline Liderazgo & 2,34 & 2,50 & 2,31 & 2,26 & 2,36 \\
\hline Conocimiento de otras culturas y costumbres & 3,20 & 2,64 & 2,92 & 2,68 & 2,65 \\
\hline Iniciativa y espiritu emprendedor & 2,83 & 3,03 & 2,83 & 2,96 & 2,96 \\
\hline Motivación por la calidad & 3,05 & 2,97 & 2,95 & 2,87 & 2,89 \\
\hline Sensibilidad medioambiental & 2,56 & 2,75 & 2,48 & 2,46 & 2,73 \\
\hline
\end{tabular}


Los datos de la tabla permiten hacer algunos comentarios. Las tres competencias más valoradas son: la capacidad de comunicación oral y escrita en lengua materna; la capacidad de organización y planificación; la capacidad para el reconocimiento de la diversidad y la multiculturalidad. Las dos primeras son competencias instrumentales, que destacan el alto valor de la comunicación y del lenguaje para un maestro, así como el reconocimiento a la planificación y control en su actividad. La tercera resalta el reconocimiento a la diversidad y diferencia de la escuela de hoy, una escuela mas inclusiva que homogeneizadora. Las competencias que siguen en valoración hacen referencia a las capacidades para las relacione interpersonales y el trabajo en equipo.
Las competencias menos valoradas son: el trabajo en un contexto internacional, el liderazgo, los conocimientos de informática, la capacidad de gestión de la información y el conocimiento de una lengua extranjera. Es un poco sorprendente que en un contexto de sociedad del conocimiento y de tecnologías de la información, por una parte, y en un marco de convergencia europeo, las competencias respectivas sean tan poco valoradas. El estudio ofrece también una valoración de determinadas competencias especificas de formación disciplinar y profesional comunes a todos los perfiles de maestro, con una metodología similar a la señalada en la competencias genéricas, que se recoge en la Tabla 4.

\section{Tabla 4 - Competencias especificas comunes de los profesores}

\begin{tabular}{|c|c|c|c|c|c|}
\hline $\begin{array}{l}\text { COMPETENCIAS ESPECÍFICAS COMUNES } \\
\text { A TODOS LOS MAESTROS }\end{array}$ & $\begin{array}{l}\text { EP- } \\
\text { LE }\end{array}$ & $\begin{array}{l}\mathrm{EP}- \\
\mathrm{EF}\end{array}$ & $\begin{array}{l}\text { EP- } \\
\text { EM }\end{array}$ & $\begin{array}{l}\text { EP- } \\
\text { NEE }\end{array}$ & EI \\
\hline \multicolumn{6}{|l|}{ SABER, CONOCER, COMPRENDER } \\
\hline $\begin{array}{l}\text { 1. Capacidad para comprender la complejidad de los } \\
\text { procesos educativos: fines, funciones de la } \\
\text { educación, teorías del desarrollo y aprendizaje, } \\
\text { entorno sociocultural, ámbito institucional y } \\
\text { organizativo de la escuela, currículum }\end{array}$ & 3,26 & 3,21 & 3,24 & 3,35 & 3,32 \\
\hline $\begin{array}{l}\text { 2. Conocimiento de los contenidos a enseñar, } \\
\text { comprendiendo su singularidad epistemológica y } \\
\text { singularidad didáctica }\end{array}$ & 3,55 & 3,54 & 3,57 & 3,51 & 3,35 \\
\hline 3. Sólida formación científico-cultural y tecnológica & 3,15 & 3,10 & 3,12 & 3,12 & 2,95 \\
\hline \multicolumn{6}{|l|}{ SABER HACER } \\
\hline $\begin{array}{l}\text { 4. Respeto a las diferencias culturales y personales } \\
\text { de los alumnos y miembros de la comunidad } \\
\text { educativa }\end{array}$ & 3,23 & 3,15 & 3,11 & 3,13 & 3,25 \\
\hline
\end{tabular}

Psicol. Argum., Curitiba, v. 25, n. 50, p. 239-259, jul./set. 2007 


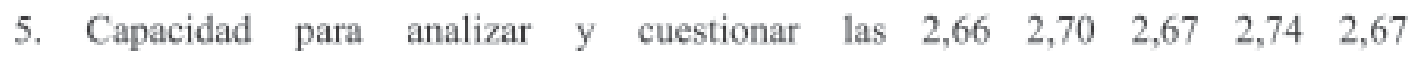
concepciones de la educación emanadas de la investigación, así como las propuestas curriculares de la Administración educativa

6. Diseño y desarrollo de proyectos educativos y $3,01 \quad 2,93 \quad 2,94 \quad 3,12 \quad 3,06$ unidades de programación, que permitan adaptar el currículum al contexto sociocultural

7. Capacidad para promover el aprendizaje autónomo $3,19 \quad 3,05 \quad 3,15 \quad 3,10 \quad 3,10$ de los alumnos, según el nivel educativo, desarrollando estrategias que eviten la exclusión y la discriminación

8. Capacidad para organizar la enseñanza de forma $2,90 \quad 2,82 \quad 2,90 \quad 2,92 \quad 2,93$ integrada, interdisciplinar, según el nivel educativo

9. Capacidad para preparar, seleccionar o construir $\begin{array}{llllll}2,96 & 2,92 & 2,97 & 3,05 & 3,18\end{array}$ materiales didácticos y utilizarlos en $\operatorname{los}$ marcos específicos de las distintas disciplinas

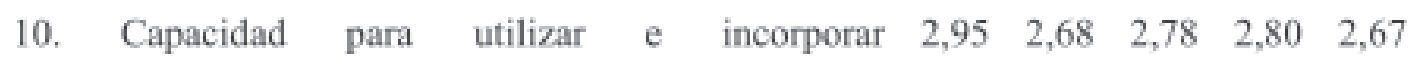
adecuadamente, en las actividades de enseñanzaaprendizaje, las tecnologías de la información y la comunicación

11. Capacidad para promover la calidad de los $2,59 \quad 2,63 \quad 2,57 \quad 2,71 \quad 2,63$ contextos de aula y centro en los que se desarrolla el proceso educativo, de modo que se garantice el bienestar de los alumnos

12. Capacidad para utilizar la evaluación en su función $3,02 \quad 3,07 \quad 3,02 \quad 2,85 \quad 3,00$ propiamente pedagógica como elemento regulador $\mathrm{y}$ promotor de la enseñanza, el aprendizaje y la propia formación

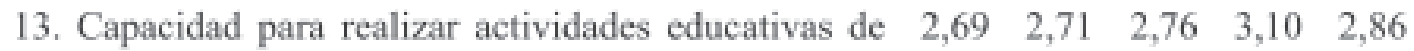
apoyo en el marco de una educación inclusiva

14. Capacidad para desempeñar la función tutorial, $3,03 \quad 3,01 \quad 3,02 \quad 3,09 \quad 3,15$ orientando a alumnos y padres 


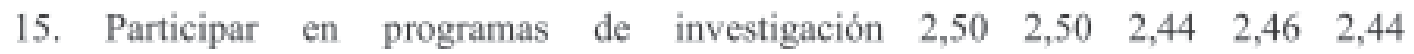
relacionados con la enseñanza y el aprendizaje, introduciendo propuestas de innovación y mejora de la calidad

\section{SABER ESTAR}

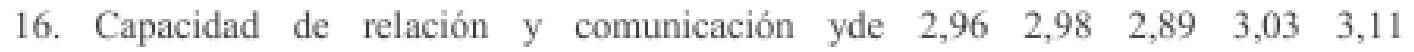
equilibrio emocional en las variadas circunstancias de la actividad profesional

17. Capacidad para trabajar en equipo con $2,89 \quad 2,90 \quad 2,91 \quad 3,06 \quad 2,96$ compañeros, compartiendo saberes y experiencias y mejorando la actividad profesional

18. Capacidad para dinamizar con el alumno la $2,92 \quad 3,01 \quad 2,90 \quad 2,85 \quad 2,95$ construcción de reglas de convivencia democrática, resolviendo los problemas y conflictos de forma colaborativa

19 Capacidad para colaborar con los distintos sectores $\begin{array}{lllll}2,52 & 2,60 & 2,54 & 2,67 & 2,62\end{array}$ de la comunidad educativa y del entorno

\section{SABER SER}

20. Tener una imagen realista d esí mismo, actuar $\begin{array}{lllll}2,89 & 2,94 & 2,90 & 2,89 & 2,85\end{array}$ conforme a las propias convicciones, asumir responsabilidades, tomar decisiones, relativizar las frustraciones.

21. Asumir la dimensión ética del maestro potenciando $\begin{array}{lllll}3,17 & 3,18 & 3,10 & 2,94 & 2,97\end{array}$ en el alumnado una actitud de ciudadanía crítica y responsable

22. Compromiso de potenciar el rendimiento $3,07 \quad 3,06 \quad 3,05 \quad 3,08 \quad 2,98$ académico de los alumnos y su progreso escolar, en el marco de una educación integral

23. Capacidad para asumir la necesidad de desarrollo $\begin{array}{llllll}2,93 & 2,93 & 2,93 & 2,85 & 2,87\end{array}$ profesional continuo, mediante la autoevaluación de la propia práctica 
Como se puede constatar los ítems más valorados son, y por este orden: 2, 1, 4, 6, 7, 14, $12,21,22$. Mientras que las competencias menos valoradas son: $5,11,15,19$. La categoría de SABER representa la de mayor interés, ya que tres de los items que la configuran ocupan los primeros lugares en importancia. Hay que destacar que el item mejor valorado, el 2 tiene que ver con el conocimiento específico de los contenidos que hay que enseñar, y que el segundo mejor valorado hace referencia al conocimiento psicosociológico que todo maestro necesita.

En la categoría de SABER HACER los items mejor valorados son el 7 , el 12 y el 14, configurando un bloque de competencias muy clásico, que hacen referencia a la capacidad de promover el aprendizaje adaptado al nivel, de saber evaluar, y de ejercer la función tutorial. También conviene destacar el item 4 , situado en el tercer nivel de importancia, y que destaca el respecto a las diferencias culturales y personales de los alumnos, cuestionando así la función uniformadora de la escuela, y expresando sensibilidad hacia la diversidad de culturas y de características personales.

En la categoría SABER SER se destacan la promoción de rendimiento académico, y también la preocupación por la educación cívica del alumno como ciudadano. De todo ello parece desprenderse que lo que más se valora es lo que concierne más directamente al trabajo de enseñanza en el aula. Y también llama la atención que los mismos académicos valoran en último lugar la investigación educativa, la capacidad de análisis crítico del currículum, la colaboración con la comunidad educativa y la mejora de los contextos de aula y centro. De lo dicho parece resaltar una visión clásica y conservadora del maestro, y una mejor consideración de lo que podríamos llamar racionalización técnica, en detrimento de una racionalidad más reflexiva y crítica, más propia del profesor investigador.

\section{Tabla 5 - Competencias especificas del Maestro de Educación Especial}

COMPETENCIAS DOCENTES ESPECIFICAS DE LOS

MAESTROS DE APOYO AL ALUMNADO CON NECESIDADES

EDUCATIVAS ESPECIALES / ESPECÍFICAS PARA

COLABORAR EN EL LOGRO DE LOS OBJETIVOS DEL

CURRICULO Y EL DESARROLLO PERSONAL DE LOS

ALUMNOS DE ED. INFANTIL, PRIMARIA Y SECUNDARIA
Sólo perfil

De

Maestro

de NEE

\section{CONOCIMIENTOS DISCIPLINARES (SABER)}

1. Conocer los fundamentos psicológicos, sociales y lingüísticos de la 3,196

diversidad de las diferentes necesidades educativas especiales/especificas, siendo capaz de asesorar tanto a los padres como a otros docentes

2. Mostrar una actitud de valoración y respeto hacia la diversidad del

alumnado, cualesquiera que fueran las condiciones o características de éste, y promover esa misma actitud entre aquellos con quienes se trabaje más directamente

3. Ser capaz de acometer, directamente o en colaboración con el resto del

profesorado, la planificación, el desarrollo y la evaluación de una respuesta educativa de calidad para el alumnado con necesidades educativas especiales/especificas, que estén asociadas a condiciones personales o sociales y cualquiera de los contextos en los que pudiera estar escolarizado 
4. Ser capaz de realizar una evaluación rigurosa de los niveles de competencia 2,582 personal del alumnado en aquellos ámbitos de su desarrollo psicosocial que puedan estar en el origen de sus necesidades especiales

5. Ser capaz de evaluar los planes de trabajo individualizados, introduciendo ajustes progresivos en los objetivos de la intervención, en la adecuación de los métodos, las pautas a seguir

6. Conocer las ayudas tecnológicas que contribuyan a mejorar las condiciones 2,716 de aprendizaje y la calidad de vida

\section{COMPETENCIAS PROFESIONALES (SABER HACER)}

7. Ser capaz de promover el desarrollo y el aprendizaje a lo largo de los 3,029 periodos de la Educación Infantil, Primaria y Secundaria, así como colaborar en el diseño de adaptaciones en Formación Profesional

8. Contribuir, a través de su acción educativa, a la mejora de la calidad de vida del alumnado de NEE, incidiendo sobre las dimensiones e indicadores que configuran dicha calidad

9. Colaborar eficazmente y de forma proactiva con los equipos de apoyo del centro o de la comunidad, especialmente con los departamentos/equipos de orientación

10. Ser capaz de evaluar la competencia curricular en las distintas áreas del currículo establecido

11. Ser capaz de determinar las necesidades educativas de los distintos alumnos, definiendo ámbitos de actuación prioritarios, así como el grado y la duración de las intervenciones, las ayudas y los poyos requeridos para promover el aprendizaje de los contenidos

12. Poder diseñar, en el marco de la programación didáctica establecida para el 2,886 conjunto de los niños y niñas del centro, planes de trabajo individualizados

13. Detectar y analizar las posibles barreras para el aprendizaje y la participación del alumnado con necesidades educativas "especiales/especificas" en el entorno del centro y en sus instalaciones, así como en su contexto 14. Participar eficazmente en procesos de mejora escolar dirigidos a introducir innovaciones que promuevan una mejor respuesta educativa a la diversidad del alumnado 
15. Buscar información de recursos existentes en la comunidad que puedan 2,629 actuar como apoyos indirectos a la tarea educativa

16. Trabajar colaboradora y cooperativamente con el resto del profesorado, los

3,448

servicios psicopedagógicos y de orientación familiar, promoviendo la mejor

respuesta educativas

Las dos competencias más valoradas, números 2 y 16, están distanciadas de las siguientes, y hacen referencia a la capacidad de mostrar una actitud de valoración y respeto hacia la diversidad del alumnado, y a promover esa misma actitud entre los profesores que trabajan en su entorno; y por otra parte, a la capacidad de trabajar colaborativamente con el resto del profesorado, con los servicios psicopedagógicos y las familias, para promover la mejor respuesta educativa. Por lo demás no hay mucha diferencia en la valoración de las competencias, y hacemos notar que las menos valoradas guardan relación con el diagnóstico educativo, tanto de la evaluación de las distintas áreas del currículo, como en la valoración de las dimensiones y características específicas de las necesidades educativas.

\section{Competencias metacognitivas. Reflexión y acción científica- técnica-ética}

La metacognición se refiere al conocimiento y control de la cognición. Versa sobre los procesos cognitivos en general, y particularmente el conocimiento que el sujeto tiene de su propio sistema mental, capacidades y limitaciones; y por otra parte, a los efectos reguladores que este conocimiento puede ejercer en su actividad. Distinguimos en la metacognición una dimensión de conocimiento y otra de control. La dimensión de conocimiento hace referencia a tres aspectos: a) persona, b) tarea y estrategia y c) contexto.
La dimensión de control la caracterizamos como a) planificación, b) supervisión y c) evaluación.

Las capacidades para reflexionar sobre nuestros propios procesos mentales tienen importantes implicaciones. Si una persona conoce sus capacidades y lo que se necesita para efectuar una ejecución eficiente en una determinada situación, entonces puede dar los pasos para satisfacer de modo adecuado esas exigencias planteadas. Sin embargo, si no es consciente de sus propias limitaciones, o de la complejidad de la tarea, difícilmente podemos esperar que adopte acciones preventivas a fin de anticipar problemas o resolverlos adecuadamente.

La metacognición entendida como control se refiere a los procesos de autorregulación utilizados por una persona en situaciones de aprendizaje y resolución de problemas. La capacidad de establecer metas y medios razonables, de determinar si se está logrando un avance satisfactorio hacia los objetivos, y de modificar debidamente la propia acción cuando el progreso no es adecuado, es otra dimensión clave de la metacognición. La metacognición entendida como regulación y control de la actividad mental se refiere a la participación activa del sujeto en el mismo proceso, esto es, antes, durante y después de realizar la actividad.

En la Figura 1 se representan los componentes del sistema mental, con la inclusión de las memorias múltiples, estrategias cognitivas, estrategias metacognitivas de conocimiento, estrategias metacognitivas de control, motivación y afectividad. 


\section{Figura 1 - Subsistemas del sistema mental}

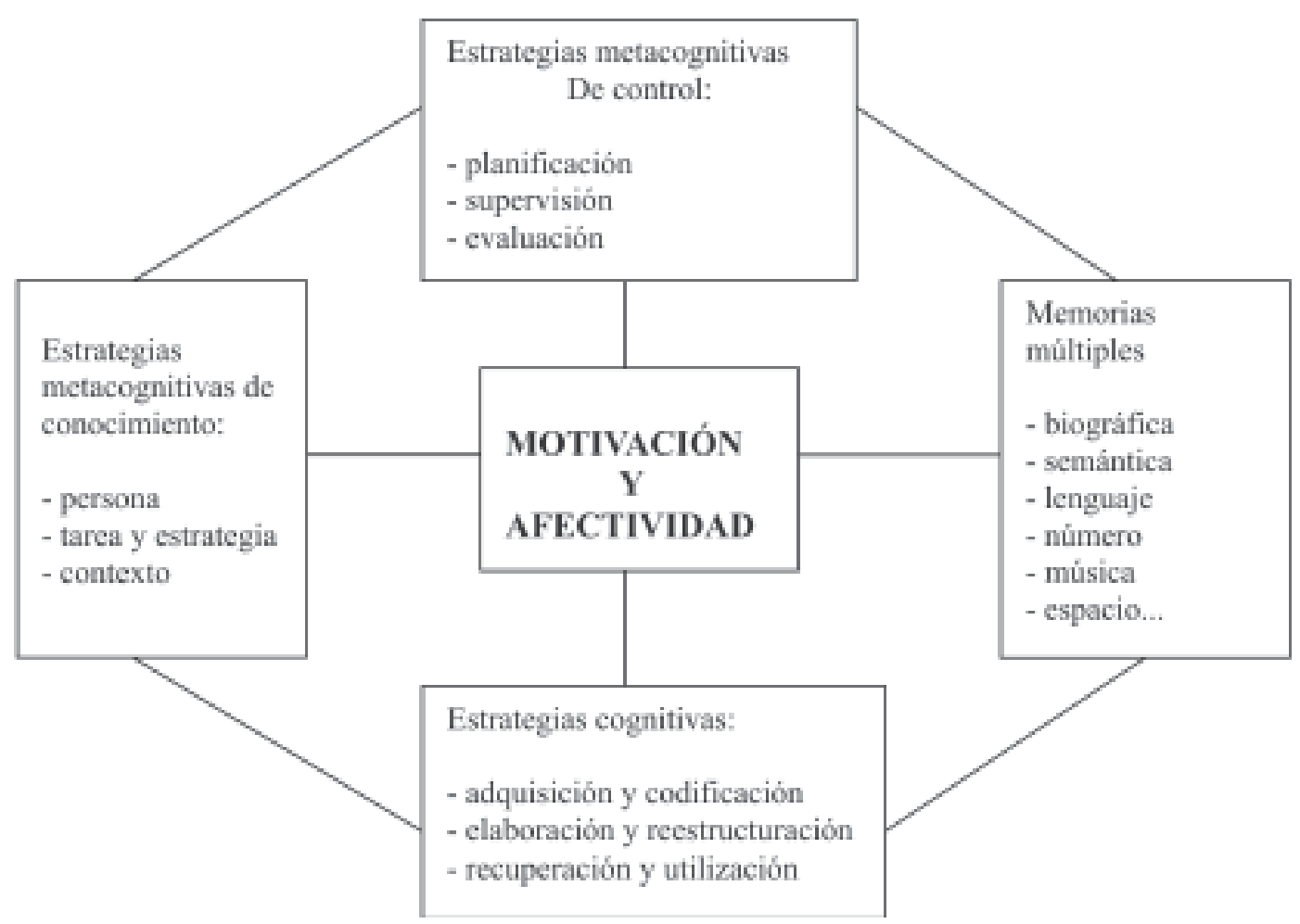

Proponemos algunas preguntas para la reflexión del profesor contextualizadas en las diversas dimensiones metacognitivas señaladas en el modelo. Metacognición como conocimiento de: a) persona, b) tarea y estrategia, c) contexto:

- Reflexiono sobre mi propia experiencia como docente?

- Reflexiono sobre aspectos prácticos, técnicos, políticos y éticos?

- Mi teoría educativa está suficientemente elaborada? Qué significa aprender, enseñar, evaluar, educar?

- Donde me sitúo en el continuum desde un enfoque formativo de carácter práctico basado en la experiencia y observación a una formación académica y especializada?

- Donde me sitúo en el continuum desde una orientación tecnológica de experto a una orientación más crítica y reflexiva?

- Donde me sitúo en el continuum desde la deficiencia-discapacidad a la minusvalía, necesidad educativa y contexto?
- Donde me sitúo en el continuum desde la profesionalización al compromiso social y comunitario?

- Presto especial atención a las diferencias personales, cognitivas, motivacionales y emocionales de los alumnos?

- Presto especial atención a las diferencias económicas y culturales de mis alumnos?

- Procuro un contexto de aprendizaje, estimulante, cooperativo y cálido?

- Mi práctica como profesor es aburrida, ilusionada, comprometida, distanciada? A continuación señalamos algunas cuestiones a plantearse desde la metacognición entendida como control: a) planificación, b) supervisión, c) evaluación:

- Mis metas en la enseñanza se centran en saber, hacer, querer, convivir, ser?

- Valoro la educación como desarrollo de la persona, ciudadano, profesional?

- Valoro la diversidad del alumnado?

- Valoro la educación como adquisición de competencias y conocimientos o como un proceso de transformación y desarrollo personal? 
- Identifico y supero obstáculos que puedo encontrar en alumnos, compañeros, familia, contexto?

- Identifico y aprovecho recursos y apoyos?

- Consigo determinar las necesidades educativas de los alumnos?

- Consigo diseñar y elaborar materiales y programas individualizados que permitan adaptar el curriculum a las diferencias personales y socioculturales de los alumnos?

- Consigo responsabilizar y comprometer al alumno en su propio proceso de aprendizaje?

- Consigo trabajar en grupo colaborativo con los profesores?

- Tengo criterios de evaluación y calidad?

- Tengo criterios para valorar la calidad de vida de los alumnos con NEE?

\section{Conclusiones}

La identificación adecuada de las competencias es requisito previo para establecer las exigencias formativas prioritarias de los futuros profesores, y por tanto para definir su curriculum de formación. En todo proyecto de reforma de la educación es necesario partir de un conocimiento, lo más riguroso posible, de la realidad social, sus recursos y limitaciones, posibilidades, fracasos y aspiraciones. Podría resultar muy interesante realizar estudios con similar perspectiva y metodología en la sociedad brasileña, para conocer la identificación y valoración de las competencias requeridas para la profesión de profesor. Estas investigaciones permitirían comparar resultados y constatar regularidades, especificidades y discrepancias. Cuando se están planteando ambiciosos proyectos de convergencia y armonización entre el Espacio Europeo de Educación Superior y el Espacio Iberoamericano del Conocimiento estos estudios resultan necesarios.

\section{Referências}

Apple M. (1986). Ideología y curriculum. Madrid: Akal.

Apple, M. (1989). Maestro y textos. Madrid: Paidos.

Arguelles, A. (1997). Formación basada en competencias laborales. Mexico: Limusa.
Burbules, N., \& Desmore, R. (1992). Los limites de la profesionalización de la docencia. Educación y Sociedad., 11, 67-73.

Delors, J. (1996). La educación encierra un tesoro. Madrid: Santillana.

Fernández Pérez, M. (1988). La profesionalización del docente. Madrid: Escuela Española.

García García, E. (1986). Función, Status y Rol del Profesor. En J. Mayor (dir.). Sociología y Psicología Social de la Educación. Madrid: Anaya.

García García, E. (1988). Condición social y feminización del profesorado de Educación Básica. Revista de Educación, 285, 249266.

García García, E. (1996). Estrés, profesión docente y personalidad del docente. Ansiedad y Estrés, 2(2-3). 245-260.

García García, E. (2004). Desarrollar las inteligencias en la sociedad del conocimiento. En Parra Luna, F. (Comp). Ante los problemas de la Universidad española: 65 propuestas para conectarla con el futuro. Madrid: Entrelíneas.

García García, E. (2006). Las competencias del profesor en la sociedad del conocimiento. En R. Mejía (Coord.). Educación, globalización y desarrollo humano. Santo Domingo, RD: Buho.

Gimeno, J. (1986). Formación de los profesores e innovación curricular. Cuadernos de Pedagogía., 139, 84-90.

Giroux, H. (1987). La formación del profesorado y la ideología del control social. Revista de Educación, 284, 53-76.

Hargreaves, D. H. (1996). Profesorado, cultura y posmodernidad. Madrid: Morata.

Hargreaves, D. H., \& Fullan, M. (1992). Understanding teacher development. Londres: Casell.

Hoyle, E. (1974). Professionality, professionalism and control in teaching. Educatonal Review, 3, 12-19.

Imbernon, F. (1994). La formación y el desarrollo profesional del profesor. Barcelona: Grao.

Kemmis, S. (1988). El curriculum más allá de la teoría de la reproducción. Madrid: Morata.

Levy- Leboyer, C. (2000). Gestión de competencias. Barcelona: Gestión, 2000. 
Marchesi, A., \& Martin, E. (2000). Calidad de la enseñanza en tiempos de cambio. Madrid: Alianza.

Ortega, F., \& Varela, A. (1991). La profesión de maestro. Madrid: CIDE.

Schon, D. (1992). La formación de profesionales reflexivos. Madrid: Paidos-MEC.

Stenhouse, L. (1982). Investigación y desarrollo del curriculum. Madrid: Morata.

Stenhouse, L. (1987). La investigación como base de la enseñanza. Madrid: Morata.

Libro Blanco. (2005). Titulo de Grado en Magisterio. Madrid: ANECA.

Tuning Educational Structures in Europe (2003). Informe final. Fase I. Deusto, Groningen: Universidades.

Recebido em: 30/05/2007

Received in: 05/30/2007

Aprovado em: 30/08/2007

Approved in: 08/30/2007 